\title{
Determinants of Paramedic Response Readiness FOR CBRNE THREATS
}

Garry Stevens, Alison Jones, George Smith, Jenny Nelson, Kingsley Agho, Melanie Taylor, and Beverley Raphael

Paramedics play a pivotal role in the response to major emergencies. Recent evidence indicates that their confidence and willingness to respond to chemical, biological, radiological, nuclear, and explosives-related (CBRNE) incidents differs from that relating to their "routine" emergency work. To further investigate the factors underpinning their readiness to respond to CBRNE incidents, paramedics in New South Wales (NSW), Australia, were asked to complete a validated online survey instrument. Univariate and multivariate analyses were performed to examine associated factors determining readiness. The sample of 663 respondents was weighted to reflect the NSW paramedic population as a whole. The univariate analysis indicated that gender, length of service, deployment concern, perceived personal resilience, CBRNE training, and incident experience were significantly associated with perceived CBRNE response readiness. In the initial multivariate analysis, significantly higher response readiness was associated with male gender, university education, and greater length of service (10-15 years). In the final multivariate model, the combined effect of training/incident experience negated the significant effects observed in the initial model and, importantly, showed that those with recent training reported higher readiness, irrespective of incident experience. Those with lower concern regarding CBRNE deployment and those with higher personal resilience were significantly more likely to report higher readiness (Adjusted Relative Risk $[A R R]=0.91,95 \%$ CI: 0.84-0.99; $A R R=1.40,95 \%$ CI: 1.11-1.72, respectively). These findings will assist emergency medical planners in recognizing occupational and dispositional factors associated with enhanced CBRNE readiness and highlight the important role of training in redressing potential readiness differences associated with these factors.

$P$ ARAMEDICS HAVE AN INTEGRAL ROLE in mass casualty response. They may be called on to attend sites affected by chemical, biological, radiological, nuclear, and explosives-related (CBRNE) agents, including their calcu- lated use by terrorist groups. ${ }^{1}$ Identifying factors that support the CBRNE response readiness of paramedics will assist workforce management and development with this critical group of responders.

Garry J. Stevens, MClinPsych, Kingsley E. Agho, PhD, and Melanie R. Taylor, PhD, are all Senior Research Fellows; and Professor Beverley Raphael, MD, is a Professor, Population Mental Health \& Disasters; all are in the Disaster Response \& Resilience Research Group (DRR), School of Medicine, University of Western Sydney, Sydney, New South Wales, Australia. Alison L. Jones, MD, is Dean of the School of Medicine, University of Western Sydney. George T. Smith, BaHSC, is Director of Special Operations, Ambulance Service of New South Wales, Rozelle, New South Wales. Jenny R. Nelson, MMgt, is Manager, Counter Terrorism, NSW Police Force Counter Terrorism and Special Tactics Command, Sydney, New South Wales. 
Effective response to CBRNE terrorism and related incidents requires a ready and willing prehospital workforce. However, recent evidence indicates that this worker population may not always feel prepared, or willing, to respond to such incidents. ${ }^{2-4}$ Their reluctance could undermine the ability of the healthcare system to meet surge capacity needs anticipated for incidents of this kind. ${ }^{5}$ A recent nationally representative sample of paramedics showed that concern about infection or contamination predicted significantly lower response willingness, compared to natural disasters such as earthquakes or floods. ${ }^{2}$ Medical staff surveyed about their willingness to work in field hospitals during mass casualty incidents were less willing to do so if CBRNE agents were involved. ${ }^{6}$ Perceived risk, knowledge of the operating environment, and self-assessed capacity to provide the care required were the factors that most strongly affected willingness to work in such environments.

While safety and capability concerns have been shown to consistently affect response readiness, ${ }^{7}$ many of the issues identified are amenable to intervention. ${ }^{8}$ For example, inadequate personal protective equipment (PPE) and training have been identified as key constraints of paramedics' CBRNE preparedness and response willingness. ${ }^{2-3}$ This was illustrated during the 1991 Gulf War when Israeli responders faced the prospect of attacks with ballistic missiles potentially armed with chemical and biological warheads. While $42 \%$ of healthcare workers indicated willingness to work in this context, this increased to $86 \%$ where it was perceived that adequate "safety measures" and "protective equipment" would be provided.'

Paramedics with recent CBRNE training consistently report higher willingness to respond to a range of CBR scenarios. ${ }^{7}$ Moreover, response willingness generally increases as training quality increases. ${ }^{2,3}$ Reilly and colleagues observed that paramedics' CBRNE response "comfort level” increased with progressive levels of training - that is, awareness level, simulation, and "hands-on" practice, with the latter format associated with significantly better outcomes for biological and chemical incidents. ${ }^{3}$ It has been argued that clear risk assessment frameworks and practical information about how to protect self and family represent key training outcomes likely to increase response willingness. ${ }^{2}$

Dispositional factors such as fear or concern have also been shown to adversely affect the readiness of emergency responders, while factors such as personal resilience-that is, the capacity to adapt and respond under conditions of stress or threat - may be associated with higher readiness. Qureshi et al found that fear for self and family was the factor associated with the lowest willingness to work during disaster events among healthcare workers, who also rated biological and chemical incidents as the event types of greatest concern. ${ }^{8}$ Paton et al suggest that terrorism, and particularly its CBRNE forms, represent qualitatively different, high threat environments in which individual and team resilience is likely to be critical to the response. ${ }^{10,11}$ They propose that adaptive capacities under conditions of stress be actively identified and developed by organizations prior to critical incidents to support optimal response and post-incident adjustment and learning. In a similar vein, specific occupational factors, such as years of work experience, rank, and education level, have been associated with both positive adaptation to stress and increased risk status in first responder groups ${ }^{12,13}$ and are also examined in this study.

To support workforce development, the aim of this study was to identify demographic, occupational, and dispositional factors associated with paramedic CBRNE response readiness. A number of studies have assessed response willingness in this context in order to estimate health workforce availability, but there has been a relative neglect of the competency perceptions underpinning the actual response of these workers. ${ }^{3,7}$ Competence is critical to the overall readiness of paramedics in this context, reflecting officers' perceived ability to perform their operational role effectively ${ }^{14}$ and, consistent with self-efficacy concepts, the level of effort and persistence likely to be invested in facing such challenges. ${ }^{10}$

Accordingly, in this study "response readiness" is defined as the perceived competence to complete functional roles within CBRNE operational environments, including suspected terrorism. It was hypothesized that specific occupational factors (CBRNE incident experience and CBRNE training) and dispositional factors (lower CBRNE deployment concern and higher personal resilience) would be associated with higher CBRNE response readiness.

\section{Methods}

A general population study by Canadian researchers assessing perceived likelihood, concern, and preparedness regarding CBRNE terrorism and related incidents was the basis for the survey instrument used in this study. ${ }^{15}$ To these core items were added validated questions regarding concerns for self or family if deployed to a CBRNE incident and perceived competence to respond to CBRNE terrorism-related incidents; these were adapted from DiMaggio et al and Reilly et al, respectively. ${ }^{2,3}$ Wording in the latter item, "comfort level in responding," was changed to "feel competent to respond," to better reflect the outcome variable of interest. Incident response experience was assessed with a single question concerning any prior attendance at a scene affected by the accidental or deliberate release of any CBRNE agent/material. The final question set was established in consultation with NSW Ambulance HAZMAT trainers and specialists. Survey protocols and informed consent procedures were approved by the ethics committees of the Sydney South West Area Health Service (Protocol No. X08-0166) and the University of Western Sydney (Approval No. H5319).

This study was based on a cross-sectional design and consisted of an internet-based survey that was forwarded by e-mail to all state-based paramedic officers in New South Wales in September 2008. The target population was all 
state-employed paramedics whose role entailed active operational duties at the time of the survey. The invitation letter contained background information about the survey and an internet web link to the online instrument, which was on a secure server and provided through Survey Monkey (www.surveymonkey.com). The survey was conducted over a 4-week period between September 29 and October 31, 2008.

\section{Outcome Measurements}

Definitions of response willingness adopted in previous studies have often not directly assessed functional competence. ${ }^{2}$ To address our research questions, response readiness was selected as the outcome variable of interest in the current analysis and was based on self-rated competence to respond operationally to each of the 5 (CBRNE) terrorism scenarios-for example, "Within my work role I feel competent to respond to the effects of [chemical] terrorism."

Terrorism was selected as the index CBRNE event, as this allowed assessment of perceived competencies in relation to these agents or materials generally, as well as specific readiness associated with their deliberate use. Examples of specific agents or materials (eg, mustard agents and sarin nerve gas for chemical incidents) were also provided in the question prologues to establish a common focus and set of operational assumptions. Responses were scored on a 5point Likert scale ("not at all," "a little," "moderately," "very," and "extremely"). The "response readiness" indicator was calculated as the combined score across the 5 (CBRNE) response scenarios. Higher scores within the score range (5-25) reflected higher response readiness.

\section{Study Variables}

Demographic, occupational, and personal disposition factors were examined to determine their associations with perceived CBRNE response readiness. The demographic and occupational factors were gender, age, marital and parental status, educational qualifications, length of paramedic service, service location (metro, nonmetro), rank, recent CBRNE training (within the previous 3 years), and previous response experience with 1 or more types of CBRNE incident ("incident experience”).

Dispositional factors consisted of separately rated concern for self and concern for family if deployed to a CBRNE incident ("deployment concern") and self-rated personal resilience. Responses regarding deployment concern were dichotomized, with the responses of "very" and "extremely" combined into the indicator of interest (ie, high concern).

Personal resilience was measured using the abbreviated Connor-Davidson Resilience Scale, the CD-RISC2. ${ }^{16}$ This validated, 2 -item version of the scale measures perceptions of stress coping ability, with separate items assessing the per- ceived ability to continue to function effectively in stressful circumstances and the perceived capacity to adapt to change. The CD-RISC2 has been shown to be able to discriminate between general population samples and individuals experiencing clinical anxiety disorders and post-traumatic stress disorder (PTSD), conditions known to be associated with stress-related reductions in coping. This scale is also sensitive to increases in personal resilience associated with pharmacological treatment for PTSD, indicating that resilience is modifiable in the context of such treatment. ${ }^{16,17}$ The scale employs a 5-point response range (0-4). Based on established community norms, ${ }^{16}$ an individual's summed scores in the range of 7-8 were regarded as representing high personal resilience in the current study, while scores of 0-6 are consistent with experiencing low/moderate levels of personal resilience.

\section{Data Analysis}

All state-employed paramedics in New South Wales were invited to participate in the survey. The precise number who received questionnaires cannot be established as various members were on leave during the study period (eg, recreational leave, staff development, sick leave). Of the completed questionnaires, 663 were suitable for analysis, which represented approximately one quarter of the estimated onduty paramedic workforce with current operational duties $(2,900)$ during the study period. The survey data were weighted to adjust for gender and the 4 different NSW paramedic locations (metro, northern, western, southern), in order to reflect the NSW paramedic population as a whole.

Ratings of perceived personal competence to respond operationally to each of the 5 (CBRNE) terrorism scenarios were summed to form a response readiness score, as described above. Cronbach's alpha coefficients demonstrated that the internal consistency of each item was satisfactory (explosive/conventional, 0.93; chemical, 0.87; biological, 0.87; radiological, 0.89; and nuclear, 0.90). Exploratory data analysis was conducted using frequency distribution for categorical variables, graphs and summary statistics for continuous variables to check the normality of the data. Preliminary analysis showed that the data were skewed, which violated ordinary linear regression assumptions, and therefore Poisson regression was used in the analysis.

In the initial stage, all of the demographic variables were entered into the model, and backward elimination was used to remove nonsignificant factors. In the second stage, combined indicators of incident experience and training were assessed, after controlling for significant demographic variables. In the third stage, deployment concern was assessed after adjusting for significant demographic variables and the combined indictors of incident experience and training. Personal resilience variables were entered into the final model to determine the effect of demographic, deployment concern, and the combined incident experience/training variables on the CBRNE readiness of paramedic officers. 
Survey means and univariate analyses were performed using Poisson regression analysis to determine the mean and relative risk, respectively, of each possible associated factor. This was followed by multivariate analyses, to examine the independent effect of each variable after controlling for other covariates. Relative risk and 95\% confidence intervals were determined for each associated factor. The statistical analyses were performed using STATA/MP version 10.0 (Stata Corporation, College Station, TX, 2007), and Poisson regression analyses were fitted using STATA survey commands to adjust for the probability of the 4 different NSW paramedic locations and gender. We considered $p \leq 0.05$ as statistically significant. All statistical tests were 2 -tailed.

\section{Results}

\section{Characteristics of the Population Sampled}

As summarized in Table 1 , the majority of paramedic respondents were male $(68.5 \%)$. The locations of respondents were nearly equally represented in the sample. Approximately $83 \%$ were married or partnered, and $56.3 \%$ had completed a university qualification at the bachelor's level or above. The proportion of paramedics who were in the 16- to 24 -year age category was relatively low $(2.1 \%)$, while those in the 35- to 44-year age category represented the largest respondent group (37\%). Approximately 25\% of paramedics had recently completed CBRNE training (ie, within the past 3 years), and of that group, $75 \%$ also reported CBRNE incident response experience, compared to $43 \%$ of those without recent training.

Approximately half of the respondents had children living with them at home. About half (50.5\%) reported high personal concern regarding potential deployment to a known or suspected CBRNE terrorist incident. Concern that family members could be affected by such an incident (ie, via secondary contamination or infection) was marginally higher $(51.4 \%)$. Despite these specific concerns, the majority of paramedics also reported high levels of personal resilience $(72.4 \%)$. It was noted that there were no major incidents reported in NSW during the period of the survey (late October to late November 2008), particularly regarding potential terrorist incidents or activity, which may have affected the validity of the survey results.

The mean response competence ratings and confidence intervals for each type of CBRNE terrorism incident are presented in Figure 1. Incidents involving conventional explosives were associated with moderate self-rated response

Table 1. Demographic characteristics of the paramedic sample $(N=3,589)^{\mathrm{a}}$

\begin{tabular}{|c|c|c|c|c|c|}
\hline Characteristic & $n$ & $\%$ & Characteristic & $n$ & $\%$ \\
\hline Location $^{\mathrm{b}}$ & & & Length of service in years $(n=3,578)$ & & \\
\hline Nonmetropolitan & 1,801 & 50.2 & $0-4$ & 799 & 22.3 \\
\hline Metropolitan & 1,788 & 49.8 & $5-9$ & 690 & 19.3 \\
\hline Gender & & & $10-14$ & 538 & 15.0 \\
\hline Male & 2,458 & 68.5 & $15-20$ & 344 & 9.6 \\
\hline Female & 1,131 & 31.5 & $20-24$ & 548 & 15.3 \\
\hline Age $(n=3,530)$ & & & $25+$ & 659 & 18.4 \\
\hline $16-24$ & 75 & 2.1 & Rank & & \\
\hline $25-34$ & 833 & 23.6 & Ambulance manager & 706 & 19.7 \\
\hline $35-44$ & 1,310 & 37.1 & Paramedic & 2,883 & 80.3 \\
\hline $45-54$ & 1,001 & 28.4 & Concern for self if CBRNE deployed & & \\
\hline $55+$ & 311 & 8.8 & No & 1,553 & 49.5 \\
\hline Highest formal qualification & & & Yes & 1,581 & 50.5 \\
\hline High school/technical college & 1,567 & 43.7 & Concern for family if CBRNE deployed & & \\
\hline University & 2,022 & 56.3 & No & 1,525 & 48.6 \\
\hline Children in household & & & Yes & 1,615 & 51.4 \\
\hline Yes & 1,788 & 49.8 & CBRNE/HAZMAT training/incident response & & \\
\hline No & 1,801 & 50.2 & Response and training & 679 & 18.9 \\
\hline No. people under 18 in household & & & Response without training & 1,160 & 32.3 \\
\hline 1 & 526 & 14.7 & No response but trained & 215 & 6.0 \\
\hline 2 & 2,214 & 61.7 & No response and no training & 1,535 & 42.8 \\
\hline $3-4$ & 611 & 17.0 & Personal resilience & & \\
\hline 5 or more & 131 & 3.7 & Low/moderate & 989 & 27.6 \\
\hline None & 107 & 3.0 & High & 2,600 & 72.4 \\
\hline \multicolumn{6}{|l|}{ Relationship status $(n=3,574)$} \\
\hline Married/partnered & 2,966 & 83.0 & & & \\
\hline Separated/divorced & 241 & 6.8 & & & \\
\hline Never married & 367 & 10.3 & & & \\
\hline
\end{tabular}

${ }^{a}$ Weighted total was 3,589 unless otherwise stated within brackets.

bNonmetropolitan: Northern, Western, and Southern districts, NSW; Metropolitan: Sydney metropolitan region. 
competence and were significantly higher than the ratings for all other agent/material classes, which were, in descending order; chemical and biological (which did not differ statistically), radiological, and nuclear incidents.

\section{Univariate Analyses}

Univariate analyses of CBRNE response readiness scores by demographic, occupational, and dispositional risk factor characteristics are shown in Table 2. Mean readiness scores were significantly higher in males than in females (10.7 vs 9.4), in those with recent CBRNE training compared to those without recent training (13.5 vs 9.2), and in those with CBRNE response experience compared to those without such experience (11.3 vs 8.8$)$. Training was a stronger predictor of response readiness than incident experience (Adjusted Relative Risk [ARR] 1.25 vs 1.12). Paramedics with recent training and incident experience reported significantly higher readiness than those with incident experience but without training (13.6 vs 11.0). Importantly, in the absence of CBRNE response experience, trained paramedics reported significantly higher readiness than those without training (13.2 vs 7.9).

Those with lower CBRNE deployment concern for self reported significantly higher readiness than those with high deployment concern (11.3 vs 9.8). Paramedics who had intermediate (10-15 years) and long lengths of paramedic service (greater than 20 years) reported significantly higher readiness than did those with less than 10 years' experience. Those with high personal resilience scores reported higher readiness than did those with low/moderate personal resilience scores (11.7 vs 6.4).

\section{Multivariate Analyses}

The results of the multivariate analysis are shown in Table 3. In the initial multivariate analysis, the following factors predicted significantly higher CBRNE response readiness: male gender (ARR $=0.88$, 95\% CI: 0.80-0.98), university level qualifications $(\mathrm{ARR}=1.10,95 \% \mathrm{CI}$ : 1.01-1.19), and intermediate duration paramedic work experience (ie, $10-14$ years compared to $0-9$ years $)(A R R=1.17,95 \%$ CI: 1.03-1.34).

When demographic, deployment concern, incident experience/training indicators, and the personal resilience variables were controlled for in the final multivariate model, interestingly, the effects of gender, education, and experience were no longer significant. The final multivariate analysis indicated that paramedics with CBRNE training and response experience reported significantly higher readiness than those with incident response experience only $(A R R=0.83$, CI: 0.76-0.90). Those with low concerns for themselves regarding terrorism-related CBRNE deployment had significantly higher readiness scores than those with high concerns for themselves $(A R R=0.91, C I$ : $0.84-$ $0.99)$. Those with high personal resilience reported significantly higher readiness $(A R R=1.40, C I$ : $1.24-1.52)$ than did those with low/moderate personal resilience.

\section{Discussion}

This survey provides the first Australian report on the factors associated with paramedic perceptions of response readiness for CBRNE terrorism and related incidents. The state representative sample, appropriate adjustment for

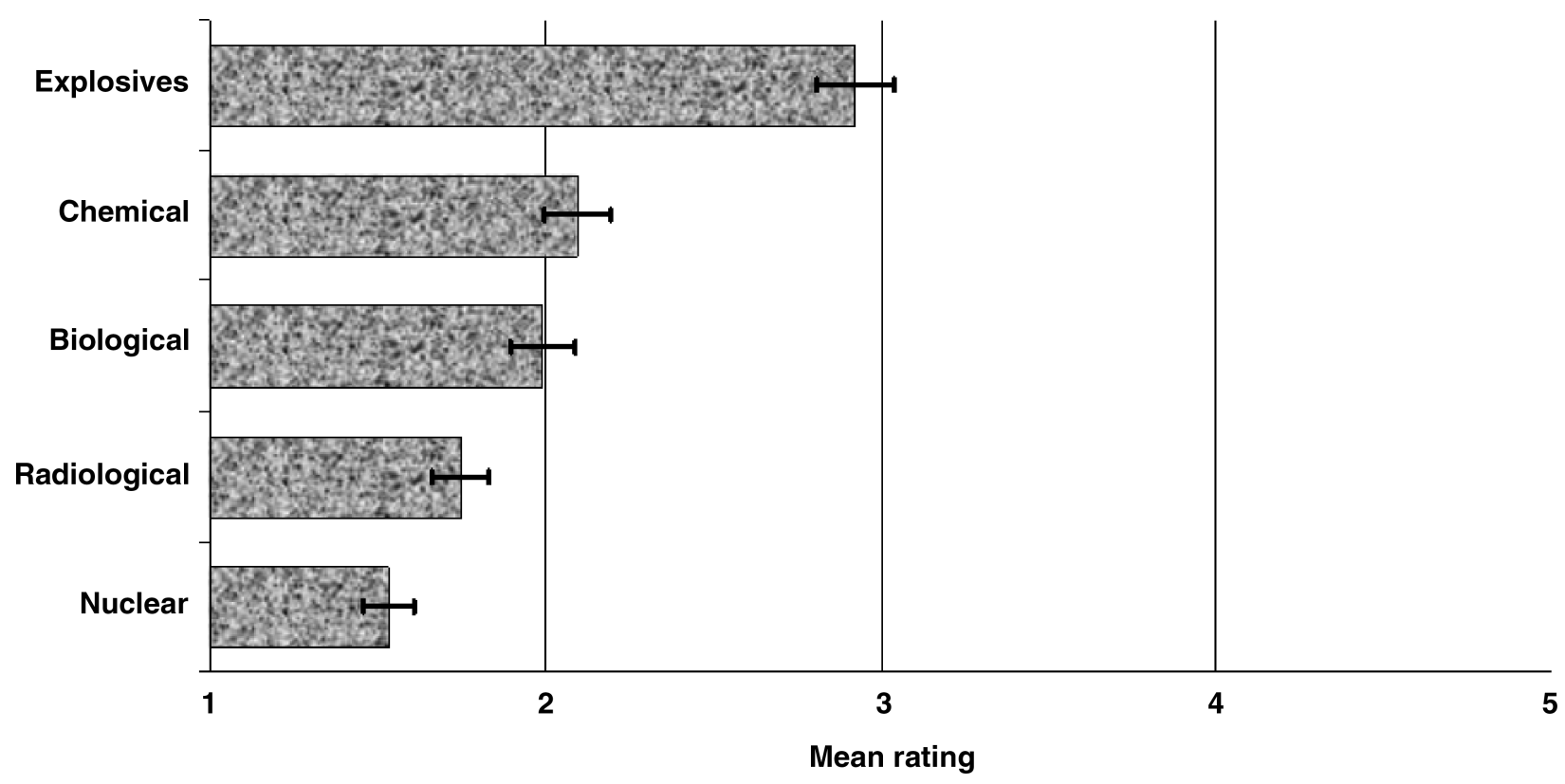

Figure 1. Weighted mean and $95 \%$ CI of perceived competence to respond to CBRNE terrorism by threat type 


\section{DETERMINANTS OF PARAMEDIC RESPONSE READINESS FOR CBRNE THREATS}

Table 2. Survey mean and univariate analyses of CBRNE response readiness by demographic, occupational, and personal disposition variables

\begin{tabular}{|c|c|c|}
\hline Characteristic & Mean & Relative Risk $(95 \% C I)^{a}$ \\
\hline \multicolumn{3}{|l|}{ Location } \\
\hline Nonmetropolitan & 10.2 & 1.00 \\
\hline Metropolitan & 10.4 & $0.95(0.85,1.06)$ \\
\hline \multicolumn{3}{|l|}{ Gender } \\
\hline Male & 10.7 & 1.00 \\
\hline Female & 9.4 & $0.88(0.80,0.97)$ \\
\hline \multicolumn{3}{|l|}{ Age } \\
\hline $16-24$ & 9.4 & 1.00 \\
\hline $25-34$ & 10.2 & $1.09(0.77,1.55)$ \\
\hline $35-44$ & 10.3 & $1.11(0.79,1.56)$ \\
\hline $45-54$ & 10.8 & $1.17(0.83,1.65)$ \\
\hline $55+$ & 10.4 & $1.13(0.78,1.64)$ \\
\hline \multicolumn{3}{|l|}{ Highest formal qualification } \\
\hline High school/technical college & 9.9 & 1.00 \\
\hline University & 10.6 & $1.07(0.99,1.15)$ \\
\hline \multicolumn{3}{|l|}{ Children in household } \\
\hline Yes & 10.4 & 1.00 \\
\hline No & 10.2 & $0.99(0.91,1.07)$ \\
\hline \multicolumn{3}{|c|}{ No. people under 18 living in household } \\
\hline 1 & 8.6 & 1.00 \\
\hline 2 & 9.3 & $1.08(0.86,1.35)$ \\
\hline $3-4$ & 10.9 & $1.26(1.03,1.54)$ \\
\hline 5 or more & 9.7 & $1.12(0.90,1.40)$ \\
\hline None & 8.1 & $0.93(0.71,1.22)$ \\
\hline \multicolumn{3}{|l|}{ Relationship status } \\
\hline Married/partnered & 10.4 & 1.00 \\
\hline Separated/divorced & 9.7 & $0.93(0.81,1.07)$ \\
\hline Never married & 9.4 & $0.90(0.79,1.02)$ \\
\hline \multicolumn{3}{|l|}{ Length of service in years } \\
\hline $0-4$ & 9.2 & 1.00 \\
\hline $5-9$ & 10.5 & $1.14(1.00,1.29)$ \\
\hline $10-14$ & 11.1 & $1.20(1.05,1.37)$ \\
\hline $15-20$ & 9.9 & $1.07(0.92,1.24)$ \\
\hline $20-24$ & 10.6 & $1.15(1.01,1.31)$ \\
\hline $25+$ & 10.8 & $1.17(1.03,1.31)$ \\
\hline \multicolumn{3}{|l|}{ Rank } \\
\hline Ambulance manager & 10.3 & 1.00 \\
\hline Paramedic & 10.3 & $1.00(0.91,1.11)$ \\
\hline \multicolumn{3}{|c|}{ Concern for self if CBRNE deployed } \\
\hline No & 11.3 & 1.00 \\
\hline Yes & 9.8 & $0.88(0.82,0.95)$ \\
\hline \multicolumn{3}{|c|}{ Concern for family if CBRNE deployed } \\
\hline No & 11.0 & 1.00 \\
\hline Yes & 10.1 & $0.92(0.84,1.02)$ \\
\hline \multicolumn{3}{|l|}{ CBRNE training } \\
\hline No & 9.2 & 1.0 \\
\hline Yes & 13.5 & $1.25(1.17,1.35)$ \\
\hline \multicolumn{3}{|l|}{ CBRNE incident response } \\
\hline No & 8.8 & 1.0 \\
\hline Yes & 11.3 & $1.12(1.01,1.25)$ \\
\hline \multicolumn{3}{|l|}{ CBRNE training/incident response } \\
\hline Response and training & 13.6 & 1.00 \\
\hline Response without training & 11.0 & $0.81(0.75,0.88)$ \\
\hline No response but trained & 13.2 & $0.97(0.86,1.09)$ \\
\hline No response and no training & 7.9 & $0.58(0.53,0.63)$ \\
\hline \multicolumn{3}{|l|}{ Personal resilience ${ }^{\mathrm{b}}$} \\
\hline Low/moderate & 6.4 & 1.00 \\
\hline High & 11.7 & $1.84(1.70,1.99)$ \\
\hline
\end{tabular}

${ }^{a}$ Relative risk ratings statistically greater than 1.00 indicate higher response readiness, and ratings statistically less than 1.00 indicate lower response readiness $(95 \%$ confidence intervals $[\mathrm{CI}]$ that include 1.00 indicate a nonsignificant result).

bersonal resilience was measured using the CD-RISC2. Values range from 0 to 8 , with 7-8 considered high personal resilience and 0-6 as low/moderate personal resilience. 
STEVENS ET AL.

Table 3. Poisson modeling of total CBRNE response readiness scores: Adjusted Relative Risk

\begin{tabular}{|c|c|c|c|c|}
\hline & Model $1^{\mathrm{a}}$ & Model $2^{\mathrm{b}}$ & Model $3^{\mathrm{c}}$ & Model $4^{\mathrm{d}}$ \\
\hline Characteristic & $R R(95 \% C I)$ & $R R(95 \% C I)$ & $R R(95 \% C I)$ & $R R(95 \% C I)$ \\
\hline \multicolumn{5}{|l|}{ Gender } \\
\hline Male & 1.00 & 1.00 & 1.00 & 1.00 \\
\hline Female & $0.88(0.80,0.98)$ & $0.95(0.87,1.04)$ & $0.93(0.85,1.02)$ & $0.95(0.87,1.04)$ \\
\hline \multicolumn{5}{|l|}{ Highest formal qualification } \\
\hline High school/technical college & 1.00 & 1.00 & 1.00 & 1.00 \\
\hline University & $1.10(1.01,1,19)$ & $1.06(0.99,1.14)$ & $1.07(0.99,1.15)$ & $1.06(0.99,1.14)$ \\
\hline \multicolumn{5}{|l|}{ Length of service in years } \\
\hline $0-4$ & 1.00 & 1.00 & 1.00 & 1.00 \\
\hline $5-9$ & $1.13(1.00,1.28)$ & $1.06(0.94,1.18)$ & $1.06(0.95,1.19)$ & $1.04(0.93,1.17)$ \\
\hline $10-14$ & $1.17(1.03,1.34)$ & $1.06(0.94,1.20)$ & $1.09(0.96,1.24)$ & $1.08(0.96,1.22)$ \\
\hline $15-20$ & $1.03(0.89,1.19)$ & $0.93(0.81,1.07)$ & $0.95(0.82,1.09)$ & $0.96(0.83,1.04)$ \\
\hline $20-24$ & $1.14(1.00,1.29)$ & $1.02(0.90,1.16)$ & $1.03(0.91,1.17)$ & $1.03(0.91,1.16)$ \\
\hline $25+$ & $1.12(0.99,1.27)$ & $1.04(0.93,1.16)$ & $1.05(0.93,1.18)$ & $1.07(0.95,1.20)$ \\
\hline \multicolumn{5}{|c|}{ CBRN/HAZMAT training/incident response } \\
\hline Response and training & & 1.00 & 1.00 & 1.00 \\
\hline Response without training & & $0.82(0.75,0.89)$ & $0.83(0.76,0.90)$ & $0.83(0.76,0.90)$ \\
\hline No response but trained & & $0.99(0.88,1.12)$ & $0.99(0.88,1.13)$ & $0.98(0.86,1.11)$ \\
\hline No response and no training & & $0.59(0.54,0.65)$ & $0.69(0.62,0.76)$ & $0.77(0.69,0.85)$ \\
\hline \multicolumn{5}{|c|}{ Concern for self if CBRNE deployed } \\
\hline No & & & 1.00 & 1.00 \\
\hline Yes & & & $0.92(0.84,1.00)$ & $0.91(0.84,0.99)$ \\
\hline \multicolumn{5}{|c|}{ Concern for family if CBRNE deployed } \\
\hline No & & & 1.00 & 1.00 \\
\hline Yes & & & $0.99(0.91,1.08)$ & $1.00(0.92,1.08)$ \\
\hline \multicolumn{5}{|l|}{ Personal resilience } \\
\hline Low/moderate & & & & 1.00 \\
\hline High & & & & $1.40(1.24,1.57)$ \\
\hline
\end{tabular}

sampling weight, and use of validated items are particular strengths of this study. The results highlight important differences in perceived CBRNE response readiness within the paramedic population associated with key occupational and dispositional risk factors.

The univariate analysis indicated that recent CBRNE training and high perceived personal resilience were the factors associated with the highest level of CBRNE response readiness. Importantly, although the multivariate analysis identified female gender, nontertiary education, and shorter length of service as risk factors for response readiness, training was found to negate these effects. Such findings are of particular practical value in that the acquisition of core response competencies, via training, is an area over which organizations have considerable control. $^{3,18}$ These results also support other recent findings that the provision, timeliness, and quality of paramedic CBRNE training is associated with both higher willingness to respond to such incidents ${ }^{2}$ and greater confidence in operational competencies. ${ }^{3}$ The latter factor, in particular, relates to the readiness measure employed in this study.
Consistent with our original hypotheses, the results of the final multivariate model showed that perceived CBRNE response readiness was predicted by the following factors: combined CBRNE response experience and recent training, lower concern for self regarding CBRNE deployment, and high personal resilience. A notable exception to the predicted relationships was that high concern for family specifically related to these deployments did not significantly affect perceived readiness.

Although training emerged as a strong predictor of readiness, the findings regarding CBRNE response experience were more equivocal. We anticipated that response experience might make an independent contribution to readiness perceptions beyond the effects of training alone, but this was not the case. Both training and experience were significant univariate factors. However, when examined as a combined indicator, CBRNE response experience added little to the overall effects of training (see Table 3).

More importantly, among those without recent training, having CBRNE incident experience did not result in significantly higher response readiness for potential CBRNE terrorist incidents. This could indicate a perception among 
these workers that knowledge gained from "routine" CBRNE incidents or alerts does not specifically enhance preparedness for CBRNE-related terrorism or, similarly, that incident debriefings do not usefully extend lessons learned to such threats.

Further examination of this issue is needed, however, to determine if the nature of CBRNE experience (unmeasured in this study) may be an important consideration. For example, higher cumulative CBRNE incident experience and/or response to particular agents or materials, in the absence of recent training, may be found to be associated with higher perceived readiness.

While CBRNE incident experience may make these operational environments more familiar, Paton et al argue that the absence of training may limit the organizing structure workers are able bring to high-threat incidents and could promote a perceived lack of control at these times. ${ }^{10}$ As noted, competency-based or hands-on training may be particularly effective, primarily because it promotes the integration of theory and application through experiential learning, consolidating skill sets through practice and feedback. ${ }^{18,19}$ Unlike real event exposure, training allows error correction and the psychomotor mastery of complex skills (eg, patient care while wearing PPE) in a safe learning environment. ${ }^{3}$ This is consistent with the specific elements of CBRNE training thought to enhance response willingness: the provision of detailed risk assessment frameworks and specific actions to protect oneself and one's family. ${ }^{2}$ Future research could identify the training modalities that are most effective in developing such competencies, including their effective components (eg, situational awareness skills, complex decision making), and also the skills development relationship between training and incident experience. One practical implication of the current findings, however, may be the preferential CBRNE deployment of personnel with recent training over those with incident experience alone.

The aims of CBRNE terrorism are inherently psychological-that is, designed to invoke fear. ${ }^{20}$ Thus, enhancing the capacity of responders to adapt to such threats is a logical form of counterterrorism. Despite this, the study of protective "dispositional" factors in this response context remains limited. ${ }^{21}$ In this study, high personal resilience and low concern for self regarding CBRNE deployment both predicted higher perceived readiness. This supports other findings showing that high perceived threat or concern predicted lower CBRNE response willingness among paramedics and healthcare workers. ${ }^{2}$ The Qureshi et al study of healthcare workers ${ }^{8}$ showed a similar result regarding deployment-related concerns for family, but an equivalent finding was not observed in this study. This may be accounted for by different exposure perceptions of paramedics and hospitalbased workers, where the latter may perceive greater exposure risk including secondary infection and contamination. It is relevant in this regard that the Qureshi study detailed findings from the 2003 SARS epidemic in Canada.

Personal resilience emerged as one of the strongest predictors of response readiness in this study. This finding indicates that an individual's perceived general capacity to adapt to stressful circumstances correlates highly with selfrated readiness for CBRNE terrorism. There is little empirical information available regarding resiliencemediated response readiness within emergency medical personnel, although Paton et al have examined these factors in recent modeling of police readiness for terrorism. ${ }^{10}$ They define resilience in this operational context as "adaptive capacity" and suggest that adaptation to novel, high-threat environments is mediated by the appraisal framework ("schema") an individual is able to employ at such times and how readily it guides interpretations and actions.

In the current study, both resilience and response readiness were perceptions reported by participants, and it is possible that the observed association reflects a common underlying construct, such as self-efficacy. To further test the current findings, a future study could assess actual or simulated incident response to determine if perceived resilience predicts demonstrated CBRNE competencies. Such a finding may have implications for recruitment and selection processes.

This study has several limitations. Our definition of "readiness" reflected the aim of assessing perceived response competence regarding CBRNE terrorism, but the extent to which this may be associated with actual CBRNE task performance remains unclear. While higher perceived competence is known to be associated with enhanced task performance in some organizational settings, ${ }^{22}$ specific evidence for this in the emergency response context remains limited. Similarly, "readiness" in this study does not specifically incorporate response willingness, which is well represented in the available literature. The cross-sectional design of this study captures only a snapshot view of the assessed indices, and no firm conclusions can be made regarding causes.

Although equal weighting was given to the 5 competence ratings (CBRNE) constituting the readiness indicator, organizations may not necessarily seek equivalent levels of preparedness across these risk scenarios. Like the current findings, other recent paramedic data show higher self-rated preparedness for conventional and chemical incidents and somewhat lower preparedness for biological and radiological/nuclear incidents, ${ }^{23}$ likely reflecting readiness priorities based on broader risk assessments. This particular finding also had the effect of lowering overall readiness ratings in the current study.

Training was considered recent if it occurred within the past 3 years. However, "residual" CBRNE training effects over longer periods (eg, 3-5 years) may have affected the current findings. As noted, it is also possible that the benefits to readiness of response experience are cumulative, which would not have been evident based on the dichot- 
omized response set for this question. Further research could explore the relative benefits of CBRNE incident experience using a more detailed measure.

It was notable that low/moderate self-rated resilience was associated with the lowest readiness score of any group. This study did not specifically assess mental health or related occupational health issues, and confounders such as clinical/subclinical depression or low job satisfaction may also have affected the current findings. ${ }^{24}$ Our primary intent was to examine general adaptive capacity (resilience) as a potential "protective" factor supporting CBRNE readiness that may be further developed. However, these preliminary findings suggest that lower levels of personal resilience represent a distinct risk factor for CBRNE response readiness and warrant further research to identify contributing factors, their effects on wider role function, and their potential remediation.

\section{Conclusion}

The results of this study support other recent findings showing that paramedic CBRNE training is associated with higher perceived readiness to respond to CBRNE terrorism and related incidents. This study adds to the existing literature detailed information about the specific effects of occupational and dispositional factors on such readiness and how factors such as training and incident experience may mediate these effects. The current findings support the suggestion that training allows responders to more readily perceive these situations as comprehensible and actionable, whereas incident experience alone may not confer such benefits. This information will assist emergency medical planners in recognizing and developing strategic and workforce factors associated with enhanced CBRNE readiness.

\section{ACKNOWLEDGMENTS}

This study was partially funded by the National Security, Science and Technology Branch of the Department of Prime Minister and Cabinet (NSST Grant No. 06-062). The analysis is part of the first author's thesis for a doctoral dissertation with the College of Health and Science at the University of Western Sydney. The authors would like to thank the Ambulance Service of New South Wales for their assistance with this study and the members of the service who participated. Our particular thanks to Peter Croft, who assisted with question development, and Gerard Azar for his technical assistance. The authors declare that they have no competing interests.

\section{REFERENCES}

1. Australian Government, Department of Foreign Affairs \& Trade. Transnational Terrorism: The Threat to Australia. 2004. http://www.dfat.gov.au/publications/terrorism/chapter2. html. Accessed December 10, 2009.
2. DiMaggio C, Markeson D, Loo GT, Redlener I. The willingess of U.S. emergency medical technicians to respond to terrorist incidents. Biosecur Bioterror 2005;3(4):331-337.

3. Reilly MJ, Markenson D, DiMaggio C. Comfort level of emergency medical service providers in responding to weapons of mass destruction events: impact of training and equipment. Prehosp Disaster Med 2007;22(4):297-303.

4. Smith E, Morgans A, Qureshi K, Burkle F, Archer F. Paramedics' perceptions of risk and willingness to work during disasters. Aust J Emerg Manage 2008;23(2):14-20.

5. Hick JL, Barbera JA, Kelen GD. Refining surge capacity: conventional, contingency, and crisis capacity. Disaster Med Public Health Prep 2009;3(Suppl 1):S59-S67.

6. Lanzilotti S, Galanais D, Leoni N, et al. Hawaii medical professionals assessment. Hawaii Med J 2002;61:162-173.

7. Chaffee M. Willingness of health care personnel to work in a disaster: an integrative review of the literature. Disaster Med Public Health Prep 2009;3:42-56.

8. Qureshi K, Gershon R, Sherman M, et al. Health care workers' ability and willingness to report to duty during catastrophic disasters. J Urban Health 2005;82(3):378-388.

9. Shapira Y, Marganitt B, Roxiner, I et al. Willingness of staff to report to their hospital duties following an unconventional missile attack: a state-wide survey. Isr Med Sci 1991;27:704-711.

10. Paton D, Violanti JM, Johnston P, Burke KJ, Clarke J, Keenan D. Stress shield: a model of police resiliency. Int J Emerg Ment Health 2008;10(2):95-107.

11. Burke KJ, Paton D. Well-being in protective services personnel: organisational influences. Australasian Journal of Disaster and Trauma Studies 2006;6. http://www.massey.ac.nz/ trauma/ issues/2006-2/burke.htm. Accessed December 10, 2009.

12. Bounds R. Factors affecting perceived stress in pre-hospital emergency medical services. Californian J Health Promot 2006;4(2):113-131.

13. Regehr C, Hill J, Knott T, Sault B. Social support, selfefficacy and trauma in new recruits and experienced firefighters. Stress and Health 2003;19;189-193.

14. Spreitzer GM. Toward a common ground in defining empowerment. Research in Organizational Change and Development 1997;10:31-62.

15. Lemyre L, Turner MC, Lee JEC, Krewski D. Public perception of terrorism threats and related information sources in Canada: implications for the management of terrorism risks. J Risk Res 2006;9(7):755-774.

16. Vaishnavi S, Connor K, Davidson J. An abbreviated version of the Connor-Davidson Resilience Scale (CD-RISC), the CDRISC2: psychometric properties and applications in psychopharmacological trials. Psychiatr Res 2007;152:293-297.

17. Connor K, Davidson J. Development of a new resilience scale: the Connor-Davidson Resilience Scale (CD-RSIC). Depress Anxiety 2003;18:76-82.

18. Markenson D, DiMaggio C, Redlener I. Preparing health professions students for terrorism, disaster, and public health emergencies: core competencies. Acad Med 2005;80(6): 517-526.

19. Blanchard PN, Thacker JW. Effective Training. 2nd ed. Englewood Cliffs, NJ: Prentice-Hall; 1999:46-50.

20. Stein BD, Tanielian TL, Eisenman DP, et al. Emotional and behavioural consequences of bioterrorism: planning a public health response. Milbank Q 2004;82(3):413-455. 


\section{DETERMINANTS OF PARAMEDIC RESPONSE READINESS FOR CBRNE THREATS}

21. Lemyre L, Corneil W, Clement J, et al. A psychosocial risk assessment and management framework to enhance response to CBRN terrorism threats and attacks. Biosecur Bioterror 2005;3(4):316-330.

22. Gist ME, Mitchell TR. Self-efficacy: a theoretical analysis of its determinants and malleability. Acad Manage Rev 1992; 17:183-211.

23. Chaput CJ, Deluhery MR, Stake CE, Martens KA, Cichon ME. Disaster training for prehospital providers. Prehosp Emerg Care 2007;11(4):458-465.

24. Regher C, Millar D. Situation critical: high demand, low control, and low support in paramedic organizations. Traumatology 2007;13(1):49-58.

Manuscript received December 11, 2009; accepted for publication May 5, 2010.

Address correspondence to:
Garry Stevens
University of Western Sydney
School of Medicine
Locked Bag 1797
Penrith South DC
New South Wales
3215 Australia

E-mail: g.stevens@uws.edu.au 